\title{
COMPATIBILITY OF LONG LIFE WITH LOW RENAL FUNCTION*
}

\section{JAMES P. O'HARE, M.D. BOSTON}

It is customary to consider chronic nephritis as a steadily progressive, degenerative process. As a rule, we do not think of the possibility of a stay in its progress when the disease has reached an advanced stage. One would certainly predict in such a case that the duration of life, with renal function near zero, must be extremely short. And yet, at the Peter Bent Brigham Hospital we have observed several patients who have lived for three or four years with such a low renal function. I wish here to report two such cases.

In the time allotted, no complete study or detailed description of the cases is possible. In order to tell as much as possible I have tried to make the chart self-explanatory.

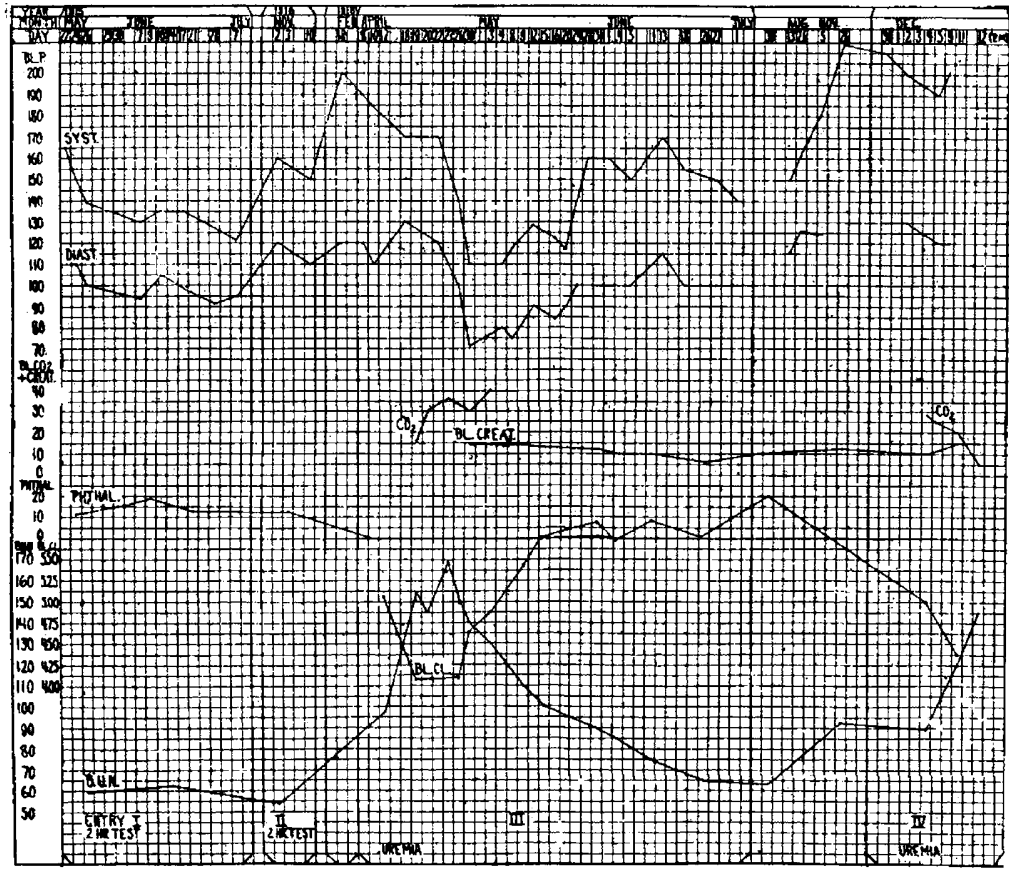

Chart 1.-Case 1: Syst., systolic blood pressure; Diast., diastolic blood pressure; $\mathrm{CO}_{2}$, bicarbonate combining power of the plasma; Bl. Creat., creatinin of the blood; $P$ hthal, phenolsulphonephthalein excretion; $N . P$. $N$. nonprotein nitrogen of the blood; $C l$., or $B l . C l$., blood chlorid; $B . U$. N., blood urea nitrogen.

\section{REPORT OF CASES}

CAsE 1.-A girl who, at 9, had scarlet fever without known sequelae; at 18 and 19 had a severe anemia necessitating her being in bed for three months and one month, respectively. In view of subsequent events I think we can fairly assume that our patient had an unrecognized acute nephritis following her scarlet fever, and at 18 and 19, acute exacerbations of a chronic nephritis. So far as we could learn, there was no other possible etiology.

About this time she began to develop vascular symptoms which took the following forms: spasmodic blurring of sight; cramps in legs and fingers, and occasional dizzy spells. Morning headaches then began to appear with increasing frequency severity and duration. In addition there were occasional drowsy spells. Apart from the latter, our patient's symptoms had been entirely vascular and not at all renal. At the age

* From the medical clinic of the Peter Bent Brigham Hospital. * Read before the Section on Practice of Medicine at the Seventiet Annual Session of the American Medical Association, Atiantic City, N.: J., June, 1919. of 23 (in May, 1915) she entered the hospital for her headaches.

Physical examination at that time revealed a moderate secondary anemia; a normal heart; no evident sclerosis of peripheral or retinal vessels; a blood pressure of 165-110; no edema, and negative eye grounds.

The urine was of low gravity, and contained a slight trace of albumin, but no casts. (It is important to note that at no time during our three and one-quarter years of observation did the urine show any sign of an active degeneration in the kidney. Hyaline casts even were very rare.) The phenolsulphonephthalein excretion was only 12 per cent., instead of the normal 60 to 80 per cent. The blood urea nitrogen was 59 mg. per hundred c.c., about four times the normal. The renal test diet showed a marked inability on the part of the kidneys to handle water, salt and nitrogen.

During the patient's six weeks' stay in the hospital, her general condition improved, the headaches were relieved, and the blood pressure fell to normal. Her renal condition, as far as we could measure it, did not change.

It would seem fair to conclude that the vascular condition with intermittent hypertension was responsible for the chief symptom-headache-and that at this time the kidneys, though markedly sclerosed, were giving her comparatively little trouble in spite of their low function. I do not believe, however, that any of us felt at the time that the patient would live for three and one-quarter years with such a low renal function.

In the sixteen months previous to her second entry in November, 1916, the patient was fairly well. Her headaches, however, returned; nosebleeds were common, and there was an occasional drowsy spell. Her symptoms were still largely vascular. She came back to the hospital chiefly for study.

This showed the following changes: (1) definite cardiac hypertrophy; (2) a return to her hypertension; (3) some sclerosis of the retinal arteries (though none could be detected in the radials and brachials), and (4) a few white spots (probably degenerative) in the retina. The urine and all of the functional tests were identical with those at the previous entry. There was no evidence of active renal degeneration, and renal function was near the minimum.

It would seem fair to conclude that the vascular disease was progressing with an effect on the heart and retina but no measurable change in the kidneys.

During 1917 the patient was not seen, but was apparently very well except for her headaches. She went about, took long walks, danced, etc. In December she had the grip, apparently without much effect. In February, 1918, she had an attack suggesting renal colic with hematuria. About the first of April she caught a severe cold, which was followed by a very severe headache, vomiting, much blurring of vision and edema of face, neck and upper sternum.

At entrance this time she showed the following new findings : (1) the tender edema mentioned above; (2) a heart of the same size but with more signs of hypertrophy; (3) definitely palpable radials; (4) greater sclerosis of retinal arteries with small hemorrhages in each eye; (5) a higher blood pressure, 190 and $120 ;(6)$ the phenolsulphonephthalein excretion had dropped to 0 , where it stayed until her death, eight months later, and her blood urea nitrogen had climbed to $98 \mathrm{mg}$.

In the hospital she began to improve rapidly. Her blood pressure began to fall. She then developed an acute infection of the antrum, and with this went immediately into uremia with drowsiness, twitching, two severe convulsions and coma. Her blood pressure did not rise and soon after showed a fall to a very low normal. There was a marked acidosis, the blood carbon dioxid reaching a level of $16 \mathrm{~mm}$. The blood urea nitrogen rose rapidly to 168 and the blood chlurid fell from 
$5.05 \mathrm{gm}$. liter to 4.20 . This drop may have been due to an infusion of sodium bicarbonate, although we know that the blood chlorid is frequently low in uremia. With the improvement in the acidosis, the blood chlorid rose to a high level. The patient then began to improve, although she was restless and twitched for three weeks. The acidosis quickly disappeared and the blood urea nitrogen fell very gradually to near its former level-70 mg. It is of interest to note that the creatinin of the blood at this time was high, $14 \mathrm{mg}$. This is supposed to indicate death within a short time, and yet the patient lived eight months. Before her discharge in July her blood pressure gradually rose, with the improvement in her general condition, to a level slightly above normal.

We can reasonably infer that at this entry her renal function, which had been sufficient up to now, had begun to give way as a result of an acute infection. She entered in impending uremia but started to improve until a new infection plunged her into an outspoken severe uremia.

In the five months previous to her last entry, Nov. 30, 1918 the patient was fairly well. Her old symptoms persisted and her blood pressure climbed rapidly to well over 200 . Then to her vascular renal symptoms she began to add cardiac ones-dyspnea and angina pectoris. There were occasional periods of low grade uremia, too, and her strength was poor. On reporting to our renal class in November, her blood urea nitrogen was found to be over $90 \mathrm{mg}$., and she was consequently sent to the hospital.

The findings at this time were similar to those at her last entry except that the radials were very tortuous; the retina showed fresh exudate and hemorrhages; blood pressure was much higher and there was twitching of the arms and legs. All of the laboratory findings were the same except that the blood urea nitrogen was higher. The blood chlorid had fallen considerably from the last estimation in July.

In the hospital she steadily went down hill. Twitching became more prominent, but there were no convulsions. Nausea and vomiting became extreme. Edema developed around her jaws. We noted a pleurisy (which was found to be superimposed on a pneumonia), and noted also what seemed to be a pericardial rub. This proved to be endocardial and due to fresh vegetations on the aortic and mitral valves. In the meantime the blood pressure had dropped some. Acidosis, as indicated by the blood carbon dioxid, developed. The blood chlorid fell (as a result either of her pneumonia or her uremia), and her blood urea nitrogen rose to $120 \mathrm{mg}$. before death.

At necropsy the kidneys were the smallest we had ever seen. The two together weighed about 60 to $65 \mathrm{gm}$. They showed a well marked vascular nephritis. The renal arteries were not abnormal except that the aortic orifices were almost closed by atheroma; but the vessels within the kidney were quite prominent. The cortex was only $2 \mathrm{~mm}$. in thickness.

Under the microscope there was practically no kidney substance left. The arteries, large and small, were all affected, showing thickening, narrowing and even obliteration of many of the smaller ones. The glomeruli were extremely rare and showed all sorts of changes from slight hyalinization of the capillary wall to complete thrombosis of the whole tuft. Some were just conpact masses covered with epithelium. The subtended tubules were mostly atrophied. There was much connective tissue. The sclerosed areas alternated with those where the glomeruli, though few, were fairly normal and connected with hypertrophied, apparently functioning, tubules.

Here, then, is presented a case of nephritis which, starting presumably from scarlet fever at 9 , had gone on for eighteen years, passing through various phases. Nine and ten years after the assumed onset the patient had what we believe to have been acute exacerbations of a chronic nephritis. Coincidently with, or subsequent to, these attacks the vascular apparatus of the body-including the kidney-began to play an everincreasing part. When the patient first came under our observation, fourteen years after the onset of the disease, she had a markedly sclerosed kidney with a very poor renal function. Just how poor this was can be readily seen. Three years before her death the two hour renal test showed very advanced disease in the kidney. Her excretion of phenolsulphonephthalein in these years has only once been as high as 18 per cent., and for the last year of her existence has been practically 0 . Her blood urea nitrogen has always been over $50 \mathrm{mg}$., about four times the average value. During the last year her blood creatinin has always been around $10 \mathrm{mg}$. And yet, in spite of this extreme renal impairment, she got along very well with these kidneys for three and one-quarter years. This was 
in January, 1908, when he was 62 years old. He had a "bad cold" followed by swelling of his eyes and face. He was in bed intermittently for eight weeks, part of the time in the Boston City Hospital. Here the records showed albuminuria and hematuria. About the first of June the edema had increased, and there were dyspnea and orthopnea. This necessitated his entry into the Massachusetts General Hospital, where he stayed for two weeks. The diagnosis of subacute glomerulonephritis, ascites, double hydrothorax and cirrbosis was made. The urine showed a large trace of albumin, a few hyaline and granular casts, and a moderate number of red and white cells. The blood pressure, determined for the first time, was found to be 150 .

The patient was not seen again for three years, but was apparently well until just before his next visit to the Massachusetts General Hospital in October, 1911. At this time the diagnosis was chronic nephritis and cardiac decompensation. His urine still showed signs of some renal activity. His blood pressure, as can be seen on the chart, had increased to $190-125$. The first phenolsulphonephthalein test showed an excretion of 17 per cent. in one hour.

The patient was seen a few times in the outpatient department of the Massachusetts General Hospital in 1911 and 1913. In 1914 he had a flareup of his nephritis following an acute diarrhea.

Shortly afterward, in April, 1914, he first entered the Brigham Hospital (Chart 2). His condition was very poor. There was an acute exacerbation of his nephritis, and uremia was impending. He was stuporous, drowsy, nauseated and had headaches. His blood pressure was over 200. His phenolsulphonephthalein excretion was 0 , and his nonprotein nitrogen climbed to $130 \mathrm{mg}$. per hundred c.c., about five times its average amount. Furthermore, his added urea and salt test showed marked inability to handle these two substances.

The outlook with such findings was without doubt grave. Certainly one could not expect him to live five more years, as he did.

He was seen a few times in 1914 in the outdoor department for headache, weakness, dyspnea and drowsiness. In March, 1915 , he entered our hospital a second time for these symptoms. He seemed to be in a state of low grade uremia. His urine was about the same, except that there was no activity. His blood pressure was still over 200 , but rather promptly fell to normal, as can be seen on the chart. The phenolsulphonephthalein had improved a bit, but was still extremely low (13 per cent.). The blood urea nitrogen was over $60 \mathrm{mg}$. Without doubt, at this time the patient's kidneys were markedly sclerosed.

He appeared at the outdoor department occasionally during 1916, 1917 and early 1918. During this time his urine showed but slight activity. His pressure varied from 225 to 160 systolic, with the diastolic more steady around 100 . The phenolsulphonephthalein excretion was only once as high as 14 per cent., and the blood urea nitrogen was between 40 and $60 \mathrm{mg}$. In the early part of 1918, a two-hour renal test showed a marked fixation of all elements.

In April, 1918, he entered the Brigham Hospital the third time for slight toxic symptoms. He was not very sick and his stay was short. His urine showed no change. His blood pressure had dropped to nearly normal, his phenolsulphonephthalein and blood urea nitrogen were about the same as before. He returned in June for two weeks because of a "convulsion" and great drowsiness. There was little new, however, to be found. His urine was no different. His blood pressure was a little higher, around 175 .

In September of that year he was admitted for the fifth time for severe dyspnea, palpitation and occasional twitching. The dyspnea was due to a chest full of fluid and was relieved by tapping. His blood pressure was again over 200 , his phenolsulphonephthalein practically 0 , and the blood urea nitrogen between 40 and $50 \mathrm{mg}$.

Between the fifth and last entries there was nothing especially new except that his heart began to give more trouble, with occasional sudden dyspnea and tightness in his chest. In November, definite alternation of the pulse was noted.

Dec. 21, 1918, he entered for the sixth time, because of precordial distress. He did not seem awfully sick but quickly became more and more drowsy. His respirations grew gradually slower and deeper. He became irrational, semiconscious and finally comatose. Twitching of hands, feet and mouth occurred, but no convulsions. There was anuria for three days. In the meantime the blood pressure dropped, as it frequently does before death. A marked acidosis developed, the blood urea nitrogen mounted quickly to $200 \mathrm{mg}$. and the phenolsulphonephthalein was, of course, 0 .

At necropsy the kidneys were very small $(62$ and $70 \mathrm{gm}$.) red and granular. The cortex measured only 2 to $3 \mathrm{~mm}$. The renal artery and larger branches were not abnormal. Microscopically, however, the vessels within the kidney showed marked arteriosclerotic changes. Many of them showed all stages of obliteration. There was very little kidney parenchyma left. The intact glomeruli were extremely scarce. Many showed recent infarction from thrombosis of the vessels leading to them. Tubules had disappeared in great numbers. Those that were left showed degeneration of all kinds. The connective tissue was particularly prominent.

COMMENT
Here, then, is a case of chronic nephritis which, starting apparently with an acute process in 1908, had active flareups during 1911 and 1914. By the latter year the process was a very severe one with the function of the kidney practically nil. After that the activity of the degenerative process was comparatively slight.

I want especially to call attention to the fact that during these five years the phenolsulphonephthalein excretion was never over 14 per cent., and most of the time much nearer 0 , and that the blood urea nitrogen was consistently four or five times the average normal.

It is difficult to determine just why this patient survived for five years with such an extremely low function. In contrast to the carefully regulated life of the first patient, who lived within the low level set by her renal function, this patient did everything that a renal patient should not do-at least up to 1917 . He was just a tramp, who ate whatever he could get and, before 1918, drank heavily. He was, however, surprisingly free from infections which might break down what little functioning tissue remained. One must conclude that the small amount of renal tissue which the patient had during the last five years was sufficient to keep him alive until the vascular disease within the kidneys had progressed to such an extent that in the last few weeks many of the small vessels became thrombosed, and with this the glomeruli supplied were put out of commission.

These two cases, then, demonstrate the possibility of prolonged existence with an exceedingly small amount of renal tissue and an extremely low renal function. The only possible explanation is that the disease during this time was a purely vascular one. The pneumonia played directly or indirectly an important part in the death of the first patient. The thrombotic obliteration of essential glomeruli was responsible for that of the second.

It can hardly be said that the true prognosis was in any way indicated by the tests performed.

536 Commonwealth Avenue.

\section{ABSTRACT OF DISCUSSION}

Dr. Herman O. Mosenthal, New York: The cases Dr. O'Hare described are of great interest and importance. He made mention of the fact that most of us would not have expected these patients to live as long as they did and I believe we can readily agree with that statement. The problem before us is to find out what factors determine the 
prognosis in nephritis and to apply this knowledge in the treatment of our patients. The expectancy of life of the sufferer with chronic nephritis may be regarded as being measured by three possible terminations: First, factors which may be considered to be entirely extrarenal, of which heart failure, apoplexy and intercurrent infections constitute those most constantly met. It is our inability to gage their progress satisfactorily that is largely responsible for the uncertain prognosis in chronic nephritis. Second, many patients with chronic nephritis die of uremia before renal function has diminished to such a degree as to render renal insufficiency any more than a contributing factor. Often the phthalein excretion lies between 20 and 30 per cent. and the blood urea nitrogen at a level of from 40 to $50 \mathrm{mg}$. per hundred cubic centimeters of blood. It is probable that this state of affairs is a toxemia, but from what tissue this poison originates, what its nature is, and what the exact conditions are that call it into action are all open questions. The sudden rise of blood nitrogen seen in one of Dr. O'Hare's cases may possibly indicate an excessive protein destruction which I believe is characteristic of certain phases of the toxemia under discussion. Third, if all the above vicissitudes leave the subject of chronic nephritis alive he finally develops an increasing renal insufficiency to which he succumbs. This is a very prolonged process and it is almost incredible how low renal function may drop and the patient maintain at least fair health. Dr. O'Hare's report illustrates this very well. I have seen some similar cases though more of them were followed for as long a period. In another condition, polycystic kidney, in which an uncomplicated renal insufficiency develops, the same phenomenon of prolonged life with a maximal impairment of renal function is often found. As far as prognosis is concerned, it becomes. evident that only very few nephritic patients really die as the result of renal insufficiency but that most of them cease to live because of extrarenal influences.

Dr. Edward F. Welis, Chicago: Satisfactory management of the patient depends largely on taking him into full confidence as to the diagnosis, the prognosis, and the general plan of treatment. Chronic interstitial nephritis is not a disease of the kidneys alone, but the blood vessels, heart, lymphatics, and other tissues are so essentially implicated that it is impossible to separate them. It takes a profound clinician to estimate within wide limits, the excretory capacity, under stress, of the interstitial nephritic kidney; and it requires a wiser physician than most of us to state how long any one of these patients may live. I have seen patients in whom the output of urea was extremely small for many successive months, in whom, when placed on some extra strain the kidneys have passed such quantities of urea as are beyond ordinary credence. Therefore, our prognosis should be extremely circumspect. Our first examination of a chronic interstitial nephritic patient should be made with painstaking care, and accuracy. The renal capacity should be estimated as closely as possible from several points of view, as, e. g., the excretion of water, of salt, the rapidity and completeness of the passage through the circulatory system, including the lymphatic circulation, of titratable solutions, and of the output of some of the waste products of metabolism. I value highly the measurement of the capacity of the kidneys to excrete urea, and always include this as an important feature of the investigation. The dietetic, regimental and medicinal measures which we employ may, probably, assist the kidney in its functions. However, some adventitious conditions are beyond our control as, e. g., the entrance into the system of an acute infection. In my own experience these most frequently transform, by disturbing the circulatory balance, a satisfactory progress to a condition from which the patient rarely completely recovers, and is often the beginning of a more or less prolonged downward course, ending fatally. Under these circumstances the patient will become dyspneic, and apneic; dependent edema will attract attention. It is absolutely necessary for the patient to maintain a posture as nearly horizontal as possible for a prolonged period, at least six weeks.

Dr. Lewis A. Conner, New York: The second of Dr. O'Hare's cases illustrates a point which is of great practical importance. That is, that in aged people the usual standards of normal kidney function are not applicable. If we attempt to prognosticate, in the case of people of 70 or 80 years of age, in terms of the standards of renal function which we apply to younger people, we are likely to make embarrassing mistakes. In such patients very low readings of the phthalein test and very high figures for blood nitrogen, creatinin, etc. are compatable with fairly good health and with many years of life.

Dr. JAmes P. O'Hare, Boston: This type of case is a rare condition. We do not see it very often but we do see it often enough to warrant hesitancy in making a prognosis in a chronically sclerosed kidney. We have had also a few cases of the edematous chronic glomerular nephritis and the patients have lived for quite a considerable period, that is, for months, with a very low renal function, and then have died from a pneumonia. I was glad to hear Dr. Mosenthal comment on the fact that death in the nephritic is very frequently due to causes not essentially nephritic but to other conditions like cardiac failure and infection. I have often been very much disappointed in cases that we had studied for a long time to find the patient dying of an acute infection or a cardiac complication when we were looking for a pure nephritis at necropsy. It is important for all of us to make records of our observations on these cases so that if a patient does live for a longer period than we expected, we can analyze the data obtained. Most of us do not do that. In fact, there has been no opportunity to do that until now because the renal tests were unknown until a few years ago. But now we have tests which are easy to make, and it is important to use them and make observations of these patients. Bear in mind, however, that even with our functional tests we are unable at present to make accurate prognoses. We make them with a fair degree of accuracy, but that degree of accuracy is often brought about by the fact that the patients die of an intercurrent infection or of cardiac failure rather than from the kidney disease.

\section{THE TREATMENT OF CHEST INJURIES *}

\section{MARION A. BLANKENHORN, M.D. CLEVELAND}

The treatment of penetrating wounds of the chest early in the war was an unsatisfactory venture offering little prospects of development, but at the end was a very satisfactory procedure promising much in the improvement of its technic and the enlargement of its scope.

Methods of examination of a wounded chest are precise; and in contrast to the abdomen or head, the damage done by a foreign body can be estimated and the treatment indicated. Foreign bodies entering the chest usually continue moving in straight lines; they produce discoverable lesions and can be located accurately by the roentgen ray. With proper appliances and training, the surgeon can invade the thorax to make anatomic repairs and remove infecting foreign substances. The effect of various infecting organisms in chest wall, pleura and lungs is still the greatest unknown quantity of the problem and the one that has yielded the least to our efforts.

It is with little satisfaction that I review the statistical reports of the various surgeons in France and England, and with even less satisfaction that I inspect my own records during two summers in forward area hospitals and one winter at the base. There were no invariable concomitants-warfare and its wounds,

* Read before the Section on Practice of Medicine at the Seventieth Annual Session of the American Medical Association, Atlantic City,
N. J., June, 1919. 